\title{
Network Control of a PVTOL System by means of an Event-Triggered Approach
}

\author{
Argel Vega-Alonzo, José Manuel Sanzón-Parra, \\ José Fermi Guerrero-Castellanos, José Juan Téllez-Guzmán, \\ Susana Sánchez-Urrieta, and Sylvain Durand ${ }^{+}$ \\ Benemérita Universidad Autónoma de Puebla (BUAP), Faculty of Electronics, \\ Puebla, Mexico \\ fguerrero@ece.buap.mx \\ + GIPSA-lab laboratory, Control Systems Dept., SySCo team, \\ CNRS-Univ. of Grenoble, France \\ http://www.fguerrero.ece.buap.mx
}

\begin{abstract}
Event-triggered control is a resource-aware sampling strategy that updates the control value only when a certain condition is satisfied, which denotes event instants. Such a technique allows a reduction of the control's computational cost and communications demand. In this paper, an asynchronous feedback is developed for event-triggered stabilization of a PVTOL (Planar Vertical Take-Off and Landing) system wherein the control loop is closed through an Internet connection. The proposed feedback ensures asymptotic stability to the desired position. Real-time experiments are carried out in order to show the convergence of the PVTOL to the desired position as well as robustness with respect to external disturbances. Results show that the proposed strategy can reduce the number of control updates and consequently reduce the communication traffic over the network without sacrificing performance of the whole system.
\end{abstract}

Keywords: PVTOL, Network Control, Event-Trigger, LQR Control, Attitude Stabilization

\section{Introduction}

A cyber-physical system (CPS) is the integration of computation with physical processes. Embedded computer networks monitor and control the physical processes, usually with feedback loops where physical processes affect computations and vice versa. The intersection between physical and information-driven (cyber) functions represents a challenge and results in innovation [1]. For CPS, the use of digital platforms and networks emerges as an obvious trend to save space, weight and energy. However, digital implementations can result in additional challenges, like determining how frequently the control signal needs to be updated and 
Argel Vega-Alonzo, et al.

applied such that the stability properties are still guaranteed.

In this context, Network-based control has emerged recently as a topic of significant interest in the control community. The reason for this is that in many practical systems it is difficult to install the physical plant, controller, sensors and actuators in the same place, and thus signals must be transmitted from one place to another. In modern systems, these components are often connected over the network media, giving rise to the so-called networked control systems [2],[3],[4]. Consequently, the traditional periodic control design cannot be applied anymore in embedded and networked systems (with limited resources) so resource-aware implementations are required. Recent work addresses alternative frameworks where the control law is event-driven. Whereas in the classical time-triggered approach the control law is computed and updated at the same rate regardless of whether it is really required or not, the event-based paradigm relaxes the periodicity of computations and communications by calling for resources whenever they are indeed necessary. A notable example is shown in [5] where the control law is updated less frequently than with a periodic scheme while still ensuring the same performance. Typical event-detection mechanisms are functions of the variation of the state (or at least the output) of the system, like in [6], [7], [8], [9], [5], [10], [11], [12], [13]. Stabilization of linear and nonlinear systems is analyzed in [14], [15], [16], [17], where the events are related to the variation of a Lyapunov function or the time derivative of a Lyapunov function (and consequently to the state too). Although event-based control is advantageous regarding computational resources, and many works report theoretical results (about stability, convergence and performance) only few works report practical implementation [18].

Among many embedded and networked cyber-physical systems, Unmanned Aerial Vehicles (UAVs) have received growing interest in research. They may prove useful for many civilian missions such as video supervision of road traffic, surveillance of urban districts, forest fire detection or building inspection. This progress was motivated by the enormous military/civil applications of such vehicles along by the technological progress in sensors, actuators, processors, power storage devices and communication technology. The Planar Vertical Take Off and Landing aircraft (PVTOL) represents a challenging nonlinear system problem that is often considered a benchmark model in aerospace engineering to design control laws for UAVs since it can be seen as the projection of a six degree of freedom flying body into a vertical plane attached to the body [19]. Furthermore, the dynamics of the PVTOL system includes many difficulties that explain the popularity of this model such as the under-actuation (three degrees of freedom for only two controls), or the non-minimum phase property (zero dynamics that are not asymptotically stable). This system also concentrates all the difficulties of the well known Brocketts integrator (also referred to as the unicycle) that one gets by neglecting the coupling factor and the gravity. Since 
its introduction in [20], a great number of approaches have been proposed to control this peculiar system. The proposed control approaches can be classified into two families: trajectory tracking or path following control approaches $[21,22]$ and stabilization control approaches $[23,24,25,26]$. In spite of the great number of papers found in the literature, the PVTOL control problem remains an active area of research.

In the present paper, a networked control systems, where the control loop is closed over a Internet connection is addressed. A deported event-triggered controller is designed to control a PVTOL system. The update policy is driven by events dependent on the time derivative of a CLF. The idea is to show that an event-triggered scheme could reduce the number of control function calls and consequently the network communications traffic even in such a case where rotor blades have to be actively controlled. The rest of the paper is organized as follows. In Section 2, the event-based control strategy for linear systems is detailed. Section 3 introduces the PVTOL mathematical model and states the problem of the control strategy for position stabilization. Experimental results are presented in Section 4 and discussions finally conclude the paper.

\section{Event-Based Control for the Stabilization of Linear Systems}

Let us consider the linear time-invariant dynamical system

$$
\dot{x}=A x+B u
$$

with $x \in \mathbb{R}^{n}, u \in \mathbb{R}^{p}$. For sake of simplicity, null stabilization with initial time instant $t_{0}=0$ is considered. Also, by event-based feedback we mean a set of two functions, namely $i$ ) an event function $e: \mathbb{R}^{n} \times \mathbb{R}^{n} \rightarrow \mathbb{R}$ that indicates if one needs to recompute the control law (when $e \leq 0$ ) or not (when $e>0$ ) and $i$ ) a feedback function $\gamma: \mathbb{R}^{n} \rightarrow \mathbb{R}^{p}$. The solution of (1) with event-based feedback $(e, \gamma)$ starting in $x_{0}$ at $t=0$ is then defined in [16] as the solution (linear case) of the differential system.

$$
\begin{aligned}
& \dot{x}=A x+B \gamma(m) \\
& m=\left\{\begin{array}{l}
x \text { if } e(x, m) \leq 0, x \neq 0 \\
m \text { otherwise }
\end{array}\right. \\
& \text { with } \quad x(0)=x_{0} \text { and } m(0)=x_{0}
\end{aligned}
$$

Here we recall the definition of semi-uniform Minimum Sampling Interval (MSI) event-triggered control: 
Argel Vega-Alonzo, et al.

Definition 1. [16] An event-triggered feedback $(\gamma, e)$ is said to be semi-uniformly $M S I$ if for all $\delta>0$, and all $x_{0}$ in the ball of radius $\delta$ centred at the origin $\mathcal{B}(\delta)$ the inter-execution times, that is the duration between two successive events, can be below bounded by some $\underline{\tau}>0$.

With this formalization, the control value is updated each time $e$ becomes negative. Usually, one tries to design an event-based feedback such that $e$ cannot remain negative (and so the control is updated only punctually). The time instants where it is negative can therefore be considered as events and $m$ is the memory of the value of the state at the last event. In addition, one also wants two events to be separated with a non vanishing time interval to avoid the Zeno phenomenon. All these properties are encompassed in the well-defined property introduced in [16], where a well-defined event-based control is a piecewise constant control with non zero sampling intervals. In the same paper, it is proved that nonlinear systems affine in the control and admitting a Control Lyapunov Function (CLF) can be globally asymptotically stabilized by means of such event-based feedback.

Proposition 1. Consider the linear time-invariant system $\dot{x}=A x+B u$. Let $P$ be, the positive definite matrix solution of the Riccati equation

$$
A^{T} P+P A-\epsilon P B B^{T} P=-Q, Q>0
$$

Then $V(x):=x^{T} P x$ is a CLF for the system since for all $x, u=-\frac{1}{2} \epsilon B^{T} P x$ with $\in \in \mathbb{R}^{+}$renders $\dot{V}$ strictly negative for $x \neq 0$.

Proof. The proof is trivial, since it is a slight variations of the well-know Lyapunov's results [27]

Then, we have the following theorem, which is a particular case of the eventbased universal formula proposed in [16]:

Theorem 1 (Event-Based LQR Stabilization). Taking the $C L F V=x^{T} P x$ for system (1), where $P$ is a positive definite matrix solution of the Riccati equation (5), then the event-based feedback $(e, \gamma)$ defined by

$$
\begin{aligned}
& \gamma(m)=-\frac{1}{2} \epsilon B^{T} P m \\
& \begin{aligned}
e(x, m) & =(\sigma-1) x^{T}\left(A^{T} P+P A\right) x \\
& -4 \varepsilon x^{T} P B R^{-1} B^{T} P(\sigma x-m)
\end{aligned} \\
& \text { with } \quad \sigma \in] 0,1[
\end{aligned}
$$

where $m$ is defined in (3). Then the control law (6) is well-defined and the closedloop system is asymptotically stable. 
The idea behind the construction of event-based feedback (6)-(7) is to compare the time derivative of the Lyapunov function $V(x)$ in the event-based case, that is applying $\gamma(m)$, and in the classical case, that is applying $\gamma(x)$ instead of $\gamma(m)$. The event function is the weighted difference between both, where $\sigma$ is the weighted value. By construction, an event is enforced when the event function vanishes to zero, that is hence when the stability of the event-based scheme does not behave as the one in the classical case. Also, events will be more frequent with smaller $\sigma$.

Proof. The proof was given in [16] for nonlinear affine in the control systems. The particular case of linear systems is hence trivial.

\section{PVTOL Mathematical Model and Control}

In this section the system's mathematical model is presented. Afterwards, the problem statement and the control design will be shown.

\subsection{Mathematical Model}

The PVTOL aircraft considered in the present work is composed of two independent motors which produce a force and a torque on the vehicle. The main thrust, $T$, is the sum of each motor thrust which is a function of the motors' angular velocities. The roll torque, $\Gamma$, is obtained from the difference between motors' thrust $f_{1}$ and $f_{2}$, that is

$$
\begin{gathered}
T=(f 1+f 2) \\
\Gamma=l(f 1-f 2)
\end{gathered}
$$

where $l$ is the distance between the rotor and the aircraft's center of mass.

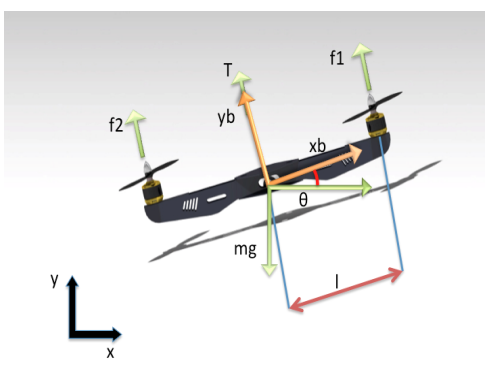

Fig. 1. PVTOL 
Argel Vega-Alonzo, et al.

From Fig. 1, we can have the following dynamic model of the PVTOL aircraft:

$$
\begin{aligned}
m \ddot{y} & =T \cos \theta-m g-c \dot{y} \\
m \ddot{x} & =-T \sin \theta-c \dot{x} \\
J \ddot{\theta} & =\Gamma-c \dot{\theta}
\end{aligned}
$$

where $x, y$ denote the horizontal and the vertical position of the aircraft's center of mass, $\theta$ is the roll angle of the aircraft with respect to the horizon, $m$ is the total mass of the aircraft, $g$ is the gravitational acceleration, $J$ is the moment of inertia and $c$ is the damping coefficient which will be considered zero for design purpose. These equations describe the motion of the vehicle as a set of three coupled second order differential equations.

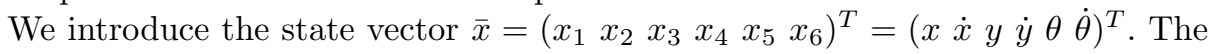
system is then represented in state variable form as

$$
\begin{aligned}
\dot{x_{1}} & =x_{2} \\
\dot{x_{2}} & =\frac{T}{m} \cos x_{5}-g-\frac{c}{m} x_{2} \\
\dot{x_{3}} & =x_{4} \\
\dot{x_{4}} & =-\frac{T}{m} \sin x_{5}-\frac{c}{m} x_{4} \\
\dot{x_{5}} & =x_{6} \\
\dot{x_{6}} & =\frac{\Gamma}{J}-\frac{c}{J} x_{6}
\end{aligned}
$$

In order to achieve the stabilization in a desired point $x_{e}$, it is convenient to redefine the state and the input so that $x_{e}$ is an equilibrium point of the system with zero input. Letting $z=\bar{x}-x_{e}, u=T-m g$, the equations become

$$
\begin{aligned}
\dot{z_{1}} & =z_{2} \\
\dot{z_{2}} & =\frac{u+m g}{m} \cos \left(z_{5}\right)-g-\frac{c}{m} z_{2} \\
\dot{z_{3}} & =z_{4} \\
\dot{z_{4}} & =-\frac{u+m g}{m} \sin \left(z_{5}\right)-\frac{c}{m} z_{4} \\
\dot{z_{5}} & =z_{6} \\
\dot{z_{6}} & =\frac{\Gamma}{J}-\frac{c}{J} z_{6}
\end{aligned}
$$

The linearization of system (12) around the origin i.e. $z \approx 0$ (actually, $x \approx$ $x_{e}$ )with $c=0$, yields

$$
\dot{z}=A z+B v
$$


where

$$
A=\left[\begin{array}{cccccc}
0 & 1 & 0 & 0 & 0 & 0 \\
0 & 0 & 0 & 0 & 0 & 0 \\
0 & 0 & 0 & 1 & 0 & 0 \\
0 & 0 & 0 & 0 & -g & 0 \\
0 & 0 & 0 & 0 & 0 & 1 \\
0 & 0 & 0 & 0 & 0 & 0
\end{array}\right], \quad B=\left[\begin{array}{cc}
0 & 0 \\
\frac{1}{m} & 0 \\
0 & 0 \\
0 & 0 \\
0 & 0 \\
0 & \frac{1}{J}
\end{array}\right]
$$

and the control vector is

$$
v=\left(\begin{array}{ll}
v_{1} & v_{2}
\end{array}\right)^{T}=(u \Gamma)^{T}
$$

\subsection{Problem Statement}

The objective is to design a control law that drives the PVTOL to a specified constant position starting from any initial condition such that the linearization remains valid.

On the other hand, the PVTOL benchmark is equipped with two computers, called local computer and deported computer, an acquisition card, an Attitude Heading Reference System (AHRS) and an infra-red sensor. The AHRS, infra-red sensor and local computer continuously monitor the state $x$ (angular and linear position and velocity) which is continuously broadcast over the network, and is denoted by $x(t)$ (see Fig. 2). Then, based on current state information and the last computed control signal, which is piecewise constant, the event-function decides when to calculate, to update and to broadcast the control signal over the network. The last step is carried out on the deported computer. Whenever the local computer receives a new control signal, it updates and applies the signals for the actuators (PWM signals).

Thus, the problem consists of showing that the PVTOL system can be stabilized by means of event-triggered feedback as defined in Section 2, i.e. with the control law (6) together with the event function (7). Another motivation is the reduction of network traffic. Reducing the traffic used for control (thanks to an event-triggered approach) allows i) to reduce traffic congestion over the network and ii) to broadcast other sensor data such as video.

\subsection{Event-Triggered Control}

Now, we have the main result.

Corollary 1. Consider the PVTOL dynamics given by (10) and (12) and the $C L F$ given by $V(z)=z^{T} P z$. Then the event-triggered feedback $(\gamma, e)$ defined by (6) with $z$ as state variable, stabilizes the PVTOL system locally $(z \rightarrow 0$ i.e. $\left.\bar{x} \rightarrow x_{e}\right)$. Furthermore, the feedback $(\gamma, e)$ is semi-uniformly MSI and smooth on $\mathbb{R}^{6}$. 
Argel Vega-Alonzo, et al.

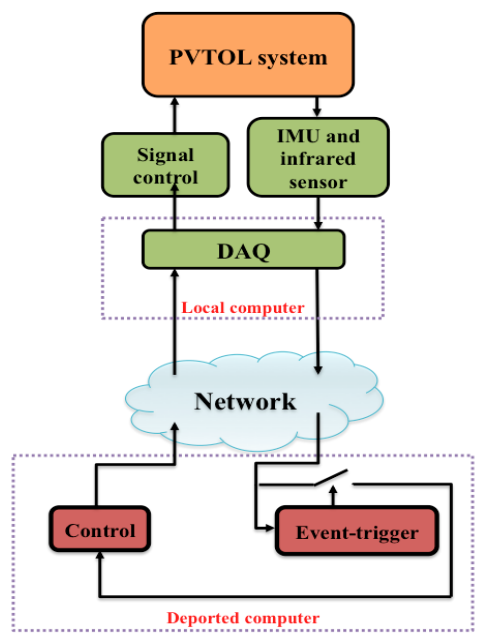

Fig. 2. Block Diagram

Remark 1. Assuming that the whole state of system (13) can be measured and $(A, B)$ is a stabilizable pair, then, it is known possible to design a state-feedback control that minimizes the value of an (infinite horizon) quadratic cost functional defined by

$$
J=\int_{0}^{\infty}\left(z^{T} Q z+\epsilon^{-1} u^{T} u\right) d t
$$

where $Q$ is a positive definite matrix and $\epsilon$ is a positive constant. The control law

$$
u=-\epsilon B^{T} P z
$$

stabilizes (13), where $P$ is a positive definite matrix solution of the Riccati equation

$$
A^{T} P+P A-\epsilon P B B^{T} P-Q=0
$$

Note that the first and second terms of (16) correspond to the energy of the controlled output and the control signal respectively, and the LQR strategy has to minimize both. However, decreasing one requires the other to be large, and viceversa. The role of $\epsilon$ consists in establishing a trade-off between these conflicting behavior (the smaller $\epsilon$ is, the larger control is and smaller the output is).

Note that the optimal LQR feedback (17) is twice the event-triggered feedback 
(6) . Therefore, the process of tuning the LQR is applicable for tuning the proposed event-based feedback which is important from a practical point of view.

\section{Experimental Results}

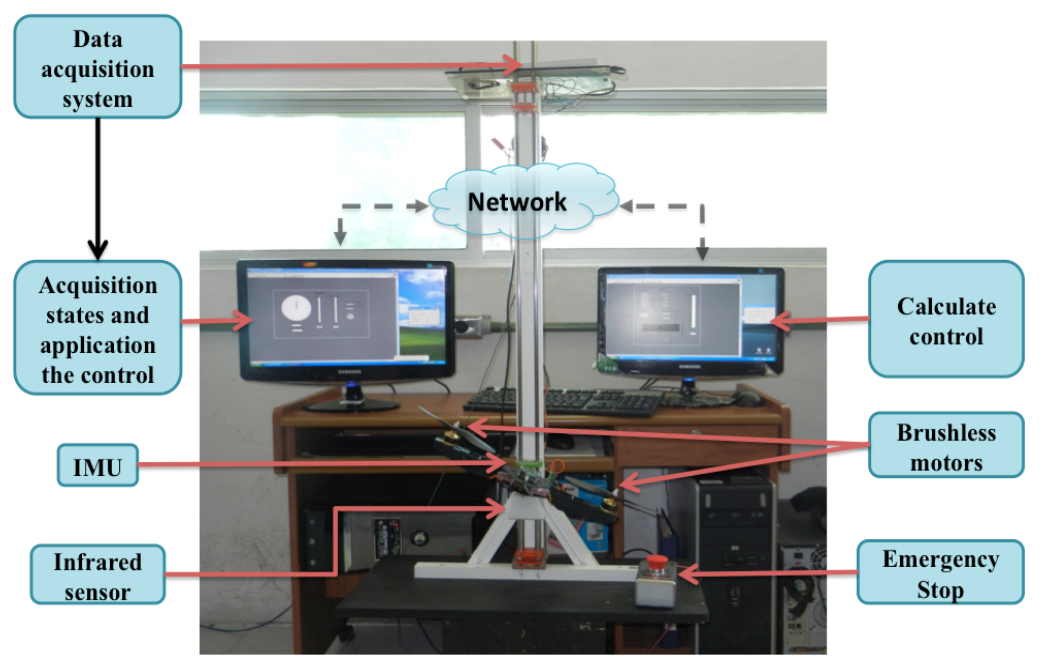

Fig. 3. System PVTOL of CCS-Lab

In this experimental section, the effectiveness of the network control is shown by means of the event-trigger approach proposed in section 2 . The real-time stabilization tests were performed with the PVTOL system developed to the Control Systems Laboratory of the Electronics Faculty at Autonomous Puebla University (see Fig. 3). The system consists of a structure of carbon fiber for the frame, two brushless motors, an inertial measurement unit ${ }^{1}$, an infrared sensor, the DAQ National Instrument ${ }^{2}$ and a mechanical structure of aluminum. The prototype is connected to a computer that acquires the state vector and applies control signals to the motors. This data is sent through the network to a second

\footnotetext{
${ }^{1}$ http://www . microstrain.com/inertial/3DM-GX1/

${ }^{2}$ http://sine.ni.com/nips/cds/view/p/lang/en/nid/203224/
} 
Argel Vega-Alonzo, et al.

computer that calculates and returns the control signals (see Fig. 3). For this, both computers have the software Labview installed. In order to hover, the propellers generate enough force for elevation. One propeller rotates clockwise and the other one counterclockwise to create torque around the $y$ axis. The rotational movement depends on the velocity difference between the two propellers.

Table 1 lists the main parameters associated with the PVTOL system.

Table 1. Parameters associated with the PVTOL

\begin{tabular}{clc}
\hline Symbol Description & Value \\
\hline$m$ & Mass of the system & $0.433 \mathrm{~kg}$ \\
\hline$d$ & $\begin{array}{l}\text { Distance from pivot to each mo- } \\
\text { tor }\end{array}$ & $0.163 \mathrm{~m}$ \\
\hline$J$ & $\begin{array}{l}\text { Equivalent moment of inertia } \\
\text { roll }\end{array}$ \\
\hline
\end{tabular}

The charts (a)-(i) present the results obtained in the two experiments. The charts (a)-(b) shows the altitude and linear velocity whereas angle roll and angular velocity are provided in the charts (c)-(d). The charts (e)-(f) show the control signals that contains the "thrust" and the "torque" and chart (g) is the Lyapunov function, which we can see decreases while the system begin stabilized. Charts (h)-(i) give the event function and a representation of the sampling instants where 1 means the control is updated and 0 indicates that is kept constant. The event function behaves as described in equation (7).

For the control law, the values $\sigma$ and $\epsilon$ of the event function for all three cases are 0.89 and 1 respectively. The $\sigma$ value determines the frequency of events.

Stabilization of PVTOL: In the first experiment, the stabilization of the PVTOL by the networked control is tested, bringing the system from $y=0.35 \mathrm{~cm}$ to $y=0.58 \mathrm{~cm}$. The results are depicted in Fig. $4(\mathrm{a})$. The angle of the system remains stable around $\theta=0$ degrees see Fig. 4(c). In Fig. 4(i), it is shown that some large intervals without any samples exist. This test was carried out in 6.5 seconds in which the control was calculated 266 times, which is $65 \%$ of the feedback for continuous control. During this period, the Lyapunov function approaches zero, as can be seen in Fig. 4(g). Fig. 4(e) and Fig. 4(f) give the force required for stabilization.

The Robustness of network control to disturbances: The second experiment tested the robustness of the proposed control against disturbances. It started with the system in the point $y=0.35 \mathrm{~cm}$. In this first part, a 
disturbance is applied in the angle $\theta=0$. After the system was stabilized, the target altitude of $y=0.58 \mathrm{~cm}$ was set, and another disturbance was applied in the altitude. Once the prototype reaches the equilibrium, the target altitude was changed to $y=0.49 \mathrm{~cm}$ and another angular disturbance was applied, see Fig. 5(a). Fig. 5(c) shows the behavior of the $x$ axis and angular velocity during disturbances. Fig. $5(\mathrm{~g})$ shows how the Lyapunov function decreases while the system is stabilized. Likewise the event function, Fig. $5(\mathrm{~h})$, is greater than 0 when there are disturbances causing more control updates. Fig. 5(i) shows the 484 times that the algorithm was calculates in 14 seconds.

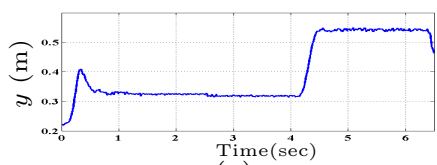

(a)

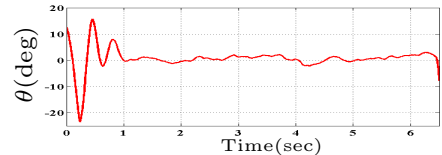

(c)

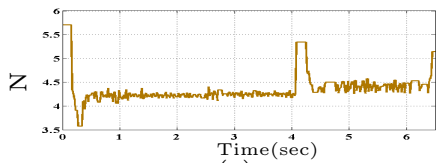

(e)

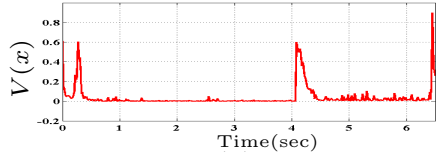

(g)

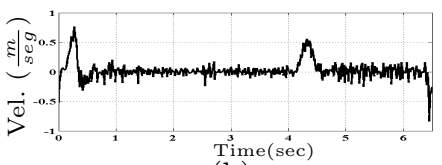

(b)

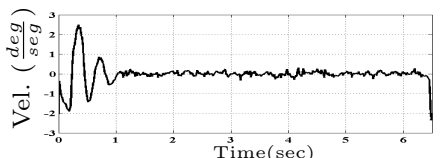

(d)

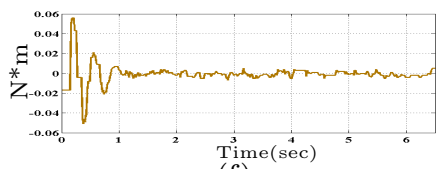

(f)

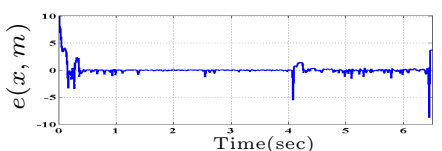

(h)

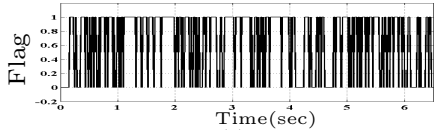

(i)

Fig. 4. Stabilization of PVTOL without disturbances 
Argel Vega-Alonzo, et al.

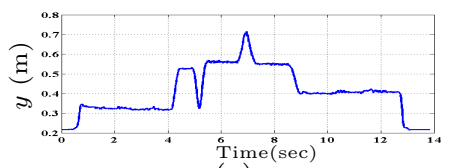

(a)

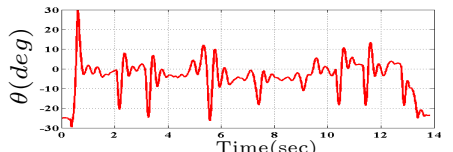

(c)

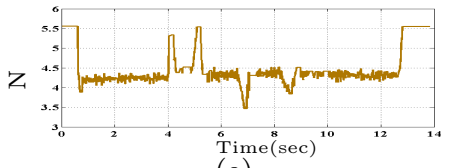

(e)

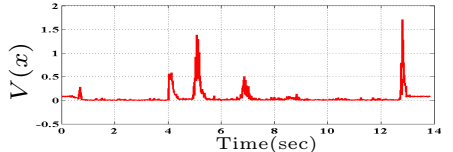

(g)

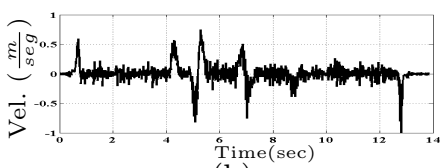

(b)

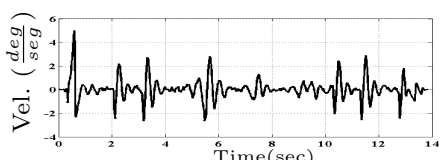

(d)

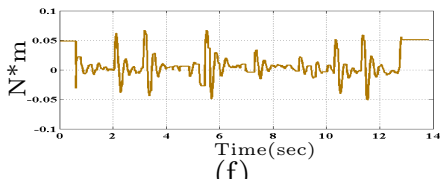

(f)

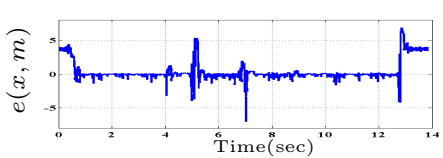

(h)

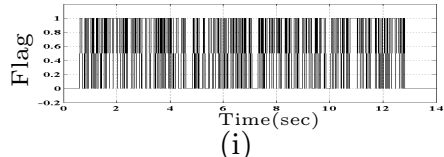

Fig. 5. Stabilization of PVTOL with disturbances 
Network Control of a PVTOL System by means of an Event-Triggered Approach

\section{Conclusions and Future Work}

The aim of this paper was to implement an event-based control strategy wherein the control loop is closed through a Internet connection. This work is based on the general formula introduced in [16] and the contributions of event-based LQR reported in [28]. The results obtained during the experiments showed the effectiveness of the proposed control strategy for the nonlinear system (although it was designed for the linearized system). The results also showed that the proposed strategy can reduce the number of control updates and consequently reduce the communication traffic over the Internet connection without sacrificing performance of the whole system. Although experiments only considered the altitude and angle, in future work the whole state will be considered.

\section{Acknowledgment}

This work is partially supported by Vicerrectoria de Investigación y Estudios de Posgrado (VIEP) de la Benemérita Universidad Autónoma de Puebla (BUAP) under grant GUCJING14-I.

\section{References}

1. Lee, E., Seshia, S.: Introduction to Embedded Systems: A Cyber-Physical Systems Approach. Lee and Seshia (2011)

2. Gao, H., Chen, T., Lam, J.: A new delay system approach to network-based control. Automatica 44 (2008) $39-52$

3. Chow, M.Y., Tipsuwan, Y.: Network-based control systems: a tutorial. In: Industrial Electronics Society, 2001. IECON '01. The 27th Annual Conference of the IEEE. (2001)

4. Park, H.S., Kim, Y.H., Kim, D.S., Kwon, W.H.: A scheduling method for networkbased control systems. Control Systems Technology, IEEE Transactions on 10 (2002) 318-330

5. Åström, K., Bernhardsson, B.: Comparison of Riemann and Lebesque sampling for first order stochastic systems. In: Proc. of the IEEE Conference on Decision and Control (CDC). (2002)

6. Årzén, K.E.: A simple event-based PID controller. In: Preprints of the 14th World Congress of IFAC, Beijing, P.R. China (1999)

7. Durand, S., Marchand, N.: Further results on event-based PID controller. In: Proc. of the European Control Conference (ECC). (2009)

8. Sandee, J., Heemels, W., van den Bosch, P.: Event-driven control as an opportunity in the multidisciplinary development of embedded controllers. In: Proc. of the IEEE American Control Conference (ACC). (2005) 1776-1781

9. Sánchez, J., Guarnes, M., Dormido, S., Visioli, A.: Comparative study of eventbased control strategies: An experimental approach on a simple tank. In: Proc. of the European Control Conference (ECC). (2009) 
Argel Vega-Alonzo, et al.

10. Heemels, W., Sandee, J., van den Bosch, P.: Analysis of event-driven controllers for linear systems. International journal of control 81 (2009) 571-590

11. Lunze, J., Lehmann, D.: A state-feedback approach to event-based control. Automatica 46 (2010) 211-215

12. Eqtami, A., Dimarogonas, D.V., Kyriakopoulos, K.J.: Event-triggered control for discrete-time systems. In: Proceedings of the IEEE American Control Conference. (2010)

13. Durand, S.: Event-based stabilization of linear system with communication delays in the measurements. Proceedings of the American Control Conference (2013)

14. Velasco, M., Martí, P., Bini, E.: On Lyapunov sampling for event-driven controllers. In: Proc. of the IEEE Conference on Decision and Control (CDC). (2009)

15. Tabuada, P.: Event-triggered real-time scheduling of stabilizing control tasks. IEEE Trans. on Automatic Control 52 (2007) 1680-1685

16. Marchand, N., Durand, S., Guerrero-Castellanos, J.: A general formula for eventbased stabilization of nonlinear systems. IEEE Transactions on Automatic Control 58 (2013) 1332-1337

17. Durand, S., Marchand, N., Guerrero Castellanos, J.F.: Event-based stabilization of nonlinear time-delay systems. In: Proceedings of the 19th World Congress of IFAC. (2014)

18. Guerrero-Castellanos, J.F., Téllez-Guzmán, J.J., Durand, S., Marchand, N., Álvarez Muñoz, J., González-Díaz, V.: Attitude stabilization of a quadrotor by means of event-triggered nonlinear control. Journal of Intelligent and Robotic Systems (2013) 1-13

19. Castillo, P., Dzul, A., Lozano, R.: Real-time stabilization and tracking of a fourrotor mini rotorcraft. IEEE Trans. on Control Sytems Technology 12(4) (2004) $510-516$

20. Hauser, J., Sastry, S., Meyer, G.: Nonlinear control design for slightly nonminimum phase systems: Application to v/stol aircraft. Automatica 28 (1992) 665-679

21. Consolini, L., Tosques, M.: On the VTOL exact tracking with bounded internal dynamics via a poincaré map approach. tac 52 (2007) 1757-1762

22. Consolini, L., Maggiore, M., Nielsen, C., Tosques, M.: Path following for the pvtol aircraft. Automatica 46 (2010) 1284-1296

23. Sira, H., Fliess, M.: Regulation of non-minimum phase outputs in a PVTOL Aircraft. In: 37th IEEE Conference on Decision and Control, Los Andes Univ., Merida, Venezuela (1998) 4222 - 4227 vol.4

24. Wood, R., Cazzolato, B., Halim, D.: A global non-linear control design for a PVTOL vehicle with aerodynamics. In: 44th IEEE Conference on Decision and Control, Adelaide, Australia (2005) 7478 - 7483

25. Francisco, R., Manzec, F., Mondié, S.: Global asymptotic stabilization of a PVTOL aircraft model with delay in the input. Springer Berlin/Heidelberg (2007)

26. Munõz, L., Santos, O., Castillo, P.: Robust nonlinear real-time control strategy to stabilize a PVTOL aircraft in crosswind. In: iros, Taipei, Taiwan (2010) 1606 1611

27. Sontag, E.D.: Mathematical control theory, deterministic finite dimensional systems. Second edn. Springler Verlag, New York Berlin Heidelberg (1998)

28. Téllez, J., Guerrero-Castellanos, J., Durand, S., Marchand, N. Lucio, R.: Eventbased lqr control for attitude stabilzation of a quadrotor. In: Latin American Control Conference (CCA). (2012) 